\title{
Malignant Cardiovascular Neoplasm
}

National Cancer Institute

\section{Source}

National Cancer Institute. Malignant Cardiovascular Neoplasm. NCI Thesaurus. Code C114940.

A primary or metastatic malignant neoplasm involving the cardiovascular system. 